\title{
Predicting Democratic Protests Paradigm from Twitter : Using Deep learning BiLSTM Model
}

This paper was downloaded from TechRxiv (https://www.techrxiv.org).

\section{LICENSE}

CC BY 4.0

SUBMISSION DATE / POSTED DATE

06-02-2022 / 11-02-2022

CITATION

Mahajan, Rhea (2022): Predicting Democratic Protests Paradigm from Twitter : Using Deep learning BiLSTM Model. TechRxiv. Preprint. https://doi.org/10.36227/techrxiv.19127435.v2

DOI

10.36227/techrxiv.19127435.v2 


\title{
Predicting Democratic Protests Paradigm from Twitter : Using Deep learning BiLSTM Model
}

\author{
Rhea Mahajan \\ Department of Computer Science \& IT \\ University of Jammu \\ Jammu and Kashmir, India \\ rhea4788@gmail.com \\ Vibhakar Mansotra \\ Department of Computer Science \& IT \\ University of Jammu \\ Jammu and Kashmir, India \\ vibhakar20@yahoo.co.in
}

\begin{abstract}
Twitter allows its users to share their concerns, ideas and daily activities online. This shared content by individuals provides a rich source of natural occurring data. The rapidity and flexibility of tweets have motivated its use as a tool for mobilizing masses for organizing strikes, protests, and other demonstrations. In this paper, we hypothesized that unrestricted data available on Twitter has potential power to predict day and location of major protests when deep learning and data mining is applied to its unstructured data. The prediction model used in the study is Bidirectional Long Short Term Memory (BiLSTM) Model. The results of our experiment shows that our model performs better than existing baselines models achieving an accuracy of $82.02 \%$ on testing data and $95.28 \%$ on training data. Overall, the aim of the paper is to study the efficacy of data mining techniques on publicly available data on Twitter in predicting mass protests and demonstrations.
\end{abstract} Mining

Keywords-Twitter, Protests, BiLSTM, Deep learning, Data

\section{INTRODUCTION}

Right to Protest is a democratic right in which citizens conduct mass demonstrations to express their views. However, due to the large number of participants, protests can sometimes result in violence and destruction. Thus, it gets important to anticipate such developing events in advance to safeguard against such repercussions. Social unrest can be defined as a public display of collective dissatisfaction or aggression by a significant human mass against a body of power such as the government or expressing a desire for change.

Social Networking Sites have changed our lives drastically and its influential and persuasive effect on the society cannot be overlooked[1].This effect has only increased and at times, does get morphed into something unpleasant and hostile, where these interactions have gravitated towards unconstructive side of things thus affecting lives of many innocuous users especially young people with very impressionable minds.. With high percolation of internet enabled mobile devices, Social Networking Sites like Twitter, Facebook, Whatsapp have been used effectively to mobilise masses for protests and demonstrations. The recent example of this is the role of these platforms in protests against the Indian agriculture acts of 2020, often referred to as the Farm Acts, passed by the Parliament of India in September 2020[2]. The farmers unions and their representatives demanded the repeal of these laws and continued to protest and demonstrate in various parts of country till Government of India relented and announced repeal of laws on November 19, 2021 [3]. In view of these protests, social media users were very active in expressing their views on the matter. The famous microblogging site, Twitter, was used extensively by protesters as a tool for planning, organizing and announcing many protests. "\#FarmersProtest" is very popular on Twitter, with thousands of users posting multiple tweets with using this hashtag and many more hashtags like this.

Using Facebook status updates, tweets from Twitter, and pictures from Instagram, you can gain insight into the social behavior of its users, and publicly available information from these platforms, especially Twitter, can provide predictive indicators of adverse law and order situations that may likely occur. Predicting protests accurately and in a timely manner will help administrative authorities take early measures rather to deal with the aftermath of potentially destructive events and will enable policy makers and administrative authorities to take proactive actions to resolve the issues.

The paper is organized as follows. After introduction Section II summaries some of the related works. Data collection and Data preprocessing techniques are explained section III. Section IV elaborates the proposed model in detail. Section V,VI and VII discusses the obtained results and simulations in detail. Finally, the study is summarized in section VIII.

\section{RELATED WORKS}

Social Networking Site, Twitter, has become an influential tool for the organization of mass gatherings of all types. However, mountains of raw data generated on these sites makes it impossible to automatically identify valuable information and patterns in real time. In last decade, many researchers have applied social media intelligence to predict civil unrest. (Crompton et al., 2013) collected publicly available tweets from Twitter, assembled similar tweets into clear, detailed and easily-interpretable audit trail which allowed end users to quickly collect information about an forthcoming civil unrest in Latin America based on successive textual and geographic filters[4]. Further (Crompton et al., 2014) applied a trained logistic regression classifier to identify accounts belonging to organizations who are likely to announce civil unrest[5]. (Cadena et al., 
2015) proposed a forecasting model [6] using a concept of activity cascades in Twitter which was originally presented by [7] to predict the occurrence of protests in Brazil, Mexico, and Venezuela. Their work was based on the assumption is that the emergence of a suitably detected activity cascade is a precursor to a real protest event that will happen on the ground. (Korolov et al., 2016) studied the possibility of predicting a social protest based on social media messaging. They considered mobilization as the precursor of participation which includes four stages: being sympathetic to the cause, being aware of the movement, motivation to take part and ability to participate. Natural Language Processing was used to create a methodology to identify mobilization in social media communication. They observed that actual occurrences of protests are preceded by growth of their probability estimated[8].

(Qiao et al., 2017) in their research used GDELT (Global Data on Events, Location, and Tone) data set to build a Hidden Markov Models (HMMs) to predict civil unrest. Their proposed method utilizes the temporal burst patterns in GDELT event streams to construct the pattern of civil unrest and to reveal the underlying mechanism for forecasting the social unrest event prediction as a sequence classification problem based on Bayes decision. The findings of their experiment with data from five countries in Southeast Asia revealed the efficiency of their framework, which performs better than logistic regression method by $7 \%$ to $27 \%$ and the baseline method by $34 \%$ to $62 \%$ [9].

(Bahrami et al., 2017) used machine learning algorithms to forecast protests. Their findings indicate that Twitter can be used as a influential tool for predicting future protests with an accuracy of $75 \%[10]$. (Dencik et al., 2018) in their study focused on three challenges: the inclusion of preexisting biases and agendas, the prominence of marketingdriven software, and the interpretation of unpredictability. Such a contextualized analysis of data uses provided important insights for the shifting terrain of possibilities for dissent[11].

Previous studies have mostly used machine learning algorithms. In this paper we have applied a deep learning based BiLSTM model to determine the polarity of protest related real-time tweets collected over a period of 33 days. The findings of our experiment shows that our model performs better than existing baselines models. We have also applied data mining to extract various patterns from our data set. Finally we have used logistic regression to establish a correlation between protest related tweets and actual protest occurrences. Overall, the aim of the paper is to study the role of social media in scenario of evolving protests.

\section{DATASET DESCRIPTION}

We ran the data collection process from 01 January 2021 to 2 February 2021 collecting around 20,000 geotagged tweets from India over a period of 33 days using Twitter search API. We searched for tweets containing the keywords "farmers", "protest", and "farmers protest". To collect geo-tagged tweets, a bounding box is used with geographic coordinates used to define the location. The Northeast and Southwest corners are selected to determine the box, and tweets geolocated within that box are collected, as well as tweets in the surrounding area based on overlapping named areas.

To extract tweets from Twitter, we must first create a Twitter account. Then, Twitter requires its users to register for an application. This application authenticates our account and provides the user with an access token and consumer key, which can be used to connect to Twitter and retrieve tweets. With Google's geo-coding API, first we obtained a bounding box in terms of latitude and longitude for each city. Then, the geo-tag filter option of Twitter's streaming API was used to extract tweets for each of those bounding boxes. The tweets were collected in JSON (Java Script Object Notation) format using tweepy, a Python library for accessing Twitter API. Fig.1. shows tweet represented in JSON format. These tweets were then stored in data frame format and were finally downloaded in CSV file format[12].

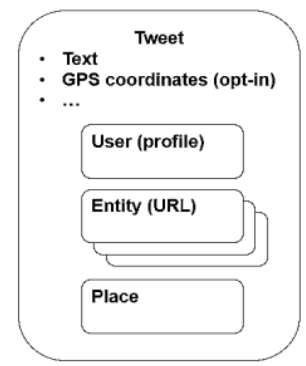

Fig. 1. Tweet represented in JSON format

\section{A. Preprocessing the Tweets}

A tweet typically contains many opinions about the data it represents. Raw tweets without preprocessing are highly unstructured containing redundant information. Preprocessing includes removal of extra spaces, URLs, emojis, conversion into lower case and converting a sentence into list of words[13]. Then tweets were clustered by geolocation .Once, the tweets were separated by location, Sentiment Intensity Analyser in NLTK( Natural Language Tool Kit) in Python was then used for labelling the tweets. Fig.2. shows protest related tweets sorted by date and location.

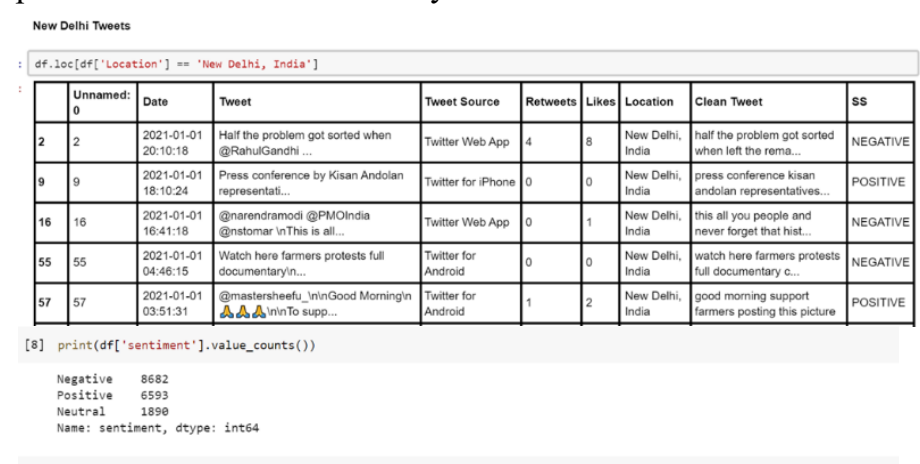

Fig. 2. Protest related Tweets sorted by date and location

The output of the Sentiment Intensity Analyser in the form of heat map and corresponding sentiment score is shown in the Fig.3., the intensity of blue colour shows the accumulated sentiment score of the Tweets on a particular day. Tweets that were categorised as Negative (dark blue) shows the probability of the protest increases before the occurrence of the actual protest. Fig.4. shows tweet count from top five cities of India. 


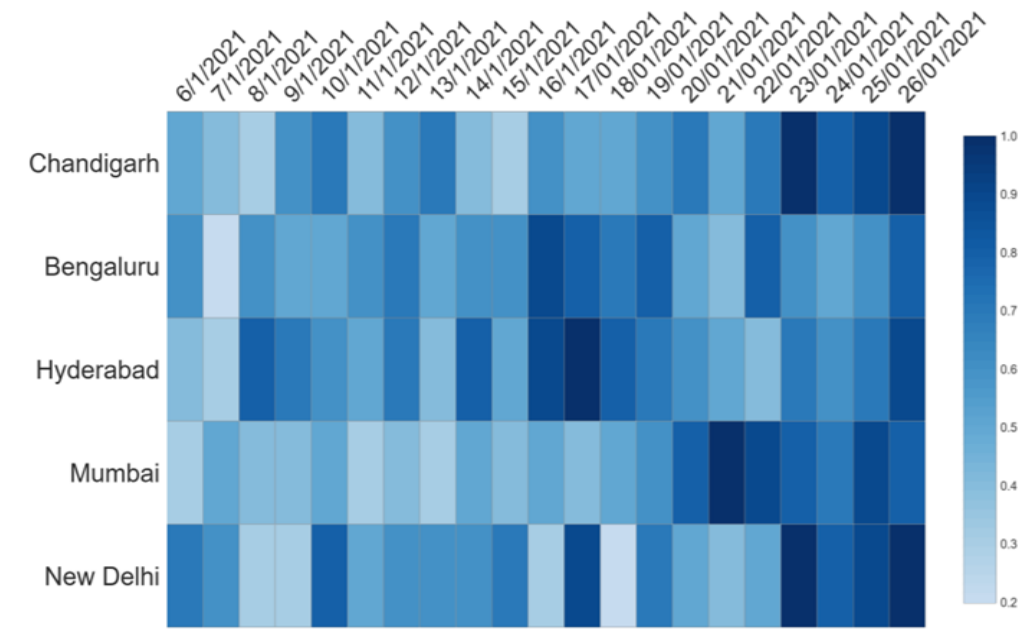

Fig. 3. Heatmap showing sentiment intensity of Protest related Tweets

\section{BILSTM MODEL}

The Sentiment analysis model used in the study is Bidirectional Long Short Term Memory (BiLSTM) Model[14]. Long-term dependencies are very complicated to understand and process for RNNs (Recurrent Neural Networks). To overcome this shortcoming, an enhanced version of the RNN, the LSTM model was developed . RNN memory can be effectively expanded by the LSTM model to capture long-term context dependencies. With this memory expansion, information may be stored in more detail for a longer period of time. The LSTM cell is called the gated cell having the ability to choose to save or discard the data. The weights allotted to the data during the exercise are used to decide whether to delete or save the information. The LSTM model consists of forget, input, and output gates. The forget gate determines whether the current information is to be retained or discarded, the input gate determines the amount of new information added to the memory, and the output gate determines whether the existing data in the cell contributes to the output. BiLSTM. is an extension of the LSTM model in which input data is processed by two LSTMs. The input sequence is first processed by the forward LSTM. This sequence is then reversed and fed to the backward LSTM. Examining the input data twice provides extra training. Raw data cannot be fed directly into a deep learning model. This data must be numerically encoded for use as an input model for deep learning. The Keras is a deep learning library which offers tools that helps you prepare raw text data. We used Keras's text preprocessing library to process each sentence into a sequence of integers. It takes each word in the sentence and substitutes it with its matching integer value from the vocabulary index. This entire sequence is then mapped to a vector of size $s$ where $s$ is the number of words in the sentence. We padded all sentences using zero padding technique so that all sentences have the identical dimension. We randomly split our data in ratio of $9: 1$, former for training and latter for testing.

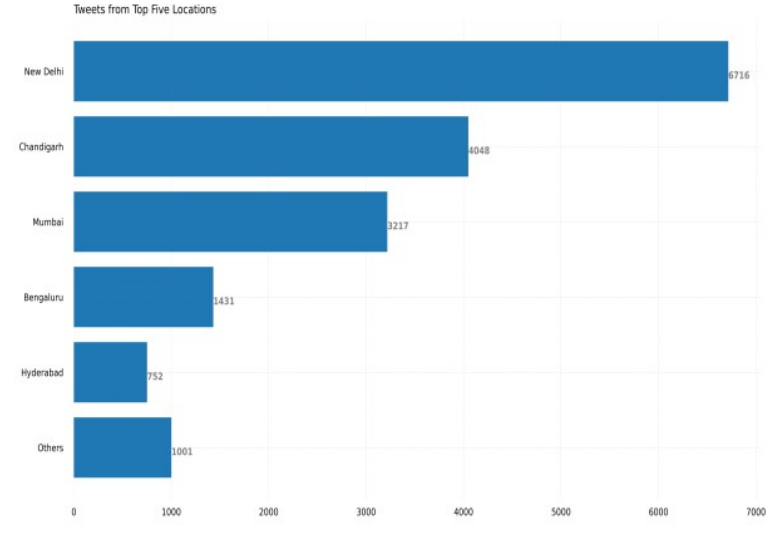

Fig. 4 . Tweets from top five cities in India

The BiLSTM model consist of two LSTMs: forward LSTM and backward LSTM. The forward LSTM takes the input and generate the forward hidden vector and backward hidden vector. These hidden vector are concatenated in aggregation layer to obtain a semantic matrix . The following equations are used

Forward LSTM equations:

$$
\begin{aligned}
& \vec{m}_{t}=\left(C_{m} \cdot x_{t}+D_{m} \cdot w_{t-1}+b_{m}\right) \\
& \vec{n}_{t}=\left(C_{n} \cdot x_{t}+D_{n} \cdot w_{t-1}+b_{n}\right) \text {, } \\
& \vec{q}_{t}=\left(C_{q} \cdot x_{t}+D_{q} \cdot w_{t-1}+b_{q}\right) \text {, } \\
& \overleftarrow{l} \sim_{t}=\left(C_{l \sim} \cdot x_{t}+D \cdot w_{t-1}+b_{l \sim}\right) \\
& \overrightarrow{l_{t}}=s_{t} \otimes l \sim_{t}+r_{t} \otimes l_{t-1}, \\
& \overrightarrow{w_{t}}=\left(l_{t}\right) \otimes u_{t} \text {. }
\end{aligned}
$$

Backward LSTM equations:

$$
\begin{aligned}
& \overleftarrow{m}_{t}=\left(C_{m} \cdot x_{t}+D_{m} \cdot w_{t+1}+b_{m}\right), \\
& \overleftarrow{n}_{t}=\left(C_{n} \cdot x_{t}+D_{n} \cdot w_{t+1}+b_{n}\right), \\
& \overleftarrow{q}_{t}=\left(C_{q} \cdot x_{t}+D_{q} \cdot w_{t+1}+b_{q}\right), \\
& \stackrel{\leftarrow}{L} \sim_{t}=\left(C_{l \sim} \cdot x_{t}+D_{l \sim} \cdot w_{t+1}+b_{l \sim}\right), \\
& \overleftarrow{l}_{t}=s_{t} \otimes l \sim \sim_{t}+r_{t} \otimes l_{t+1}, \\
& \overleftarrow{w}_{t}=\left(l_{t}\right) \otimes u_{t} .
\end{aligned}
$$

where $m, n, q$ are the forget, input, and output gates. $x_{t}$ is input state, $\mathrm{w}_{\mathrm{t}}$ is hidden state that is sent to the further LSTM layer within the network, $l_{t}$ is the current cell state, $b$ is bias, and $\mathrm{C}$ and $\mathrm{B}$ representthe weight metrics, respectively. The next later is dropout layer. Then we apply a dropout of 0.5 to prevent model from overfitting. Finally activation function softmax is applied to calculate the output probability of Tweet $t_{i}$ belonging to sentiment class $S$. The working of BiLSTM model is shown in Fig.5. The training and testing accuracy of the model for ten epochs has been plotted in graph shown in Fig.6. (a) and the training and testing loss of the model for ten epochs has been plotted in graph shown in Fig.6.(b) 


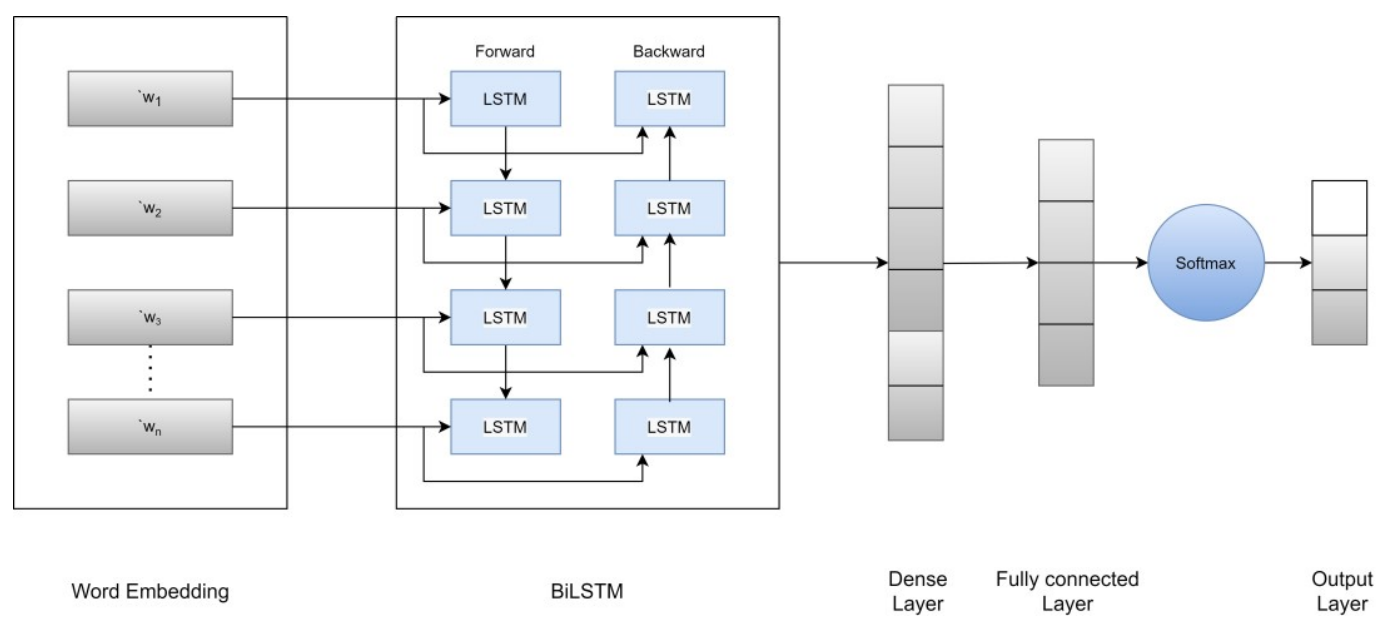

Fig. 5. Working of BiLSTM Model

\section{RESULTS}

We evaluated our proposed method on different metrices. The results show that our model using BiLSTM outperforms other existing machine learning and deep learning models[2] achieving accuracy of $82.02 \%$ on testing data and $95.28 \%$ on training data. Table 1 shows comparison of our model with other baseline models. Fig. 6 shows model loss and accuracy on testing and training data. We used 5 fold crossvalidation by training the train set five times, testing the testing set five times, and taking the average accuracy as the final result. Algorithm 1 present the pseudocode of the proposed method. Since the BiLSTM cell traverse the input text twice, the time complexity of our algorithm is $\mathrm{O}\left(\mathrm{m}^{2} \mathrm{k}^{2}\right.$ $2 c_{\text {in }} 2 c_{\text {out }}$ ) where $m$ is the size of the output graphs, $k$ is the size of the kernel, $c_{\text {in }}$ is number of units in input layer and $\mathrm{c}_{\text {out }}$ is number of units in output layer. Although, the algorithm has high time complexity but it performs well in terms of spatial complexity as BiLSTM captures global features from the text thereby reducing the dimensions of the feature vector. Furthermore, a drop out of 0.5 is applied which drops the number of trainable parameters in each iteration thereby stopping the model from over-fitting.

\section{ALGORITHM 1}

Input: Farmers protest Tweets, $\mathrm{T}_{\text {train }}, \mathrm{T}_{\text {test }}$

Output :Sentiment label: Positive,Neutral, Negative

Start

\section{Hyperparameter initialization}

$\mathrm{T}_{\text {train }}$ size $=85 \%, \mathrm{~T}_{\text {test }}$ size $=15 \%$ embedded dimension $=128$

batch size $=64$,epochs $=10$

Prepare model for fitting

For each input $t \in T_{\text {train }}$

Create embedded vector for each word in $T=\left\{\mathrm{t}_{1}, \mathrm{t}_{2} \ldots \ldots . . \mathrm{t}_{\mathrm{m}}\right\}$ Generate eigen vector forward and eigen vector backward using equation (1-12)

Obtain semantic matrix by concatenating all eigen vectors Apply a dropout $=0.5$ to prevent model from overfitting Apply activation function Softmax to calculate the output probability of Tweet $t_{i}$ belonging to sentiment class $S$.

$$
p\left(t_{i} \mid \hat{\theta}\right)=\frac{\exp \left(\beta_{i}^{T} \hat{\theta}\right)}{\sum_{j=1}^{S} \exp \left(\beta_{j}^{T} \hat{\theta}\right)}
$$

For each input $\mathrm{t} \in \mathrm{T}_{\text {test }}$

Calculate the sentiment polarity using trained model .

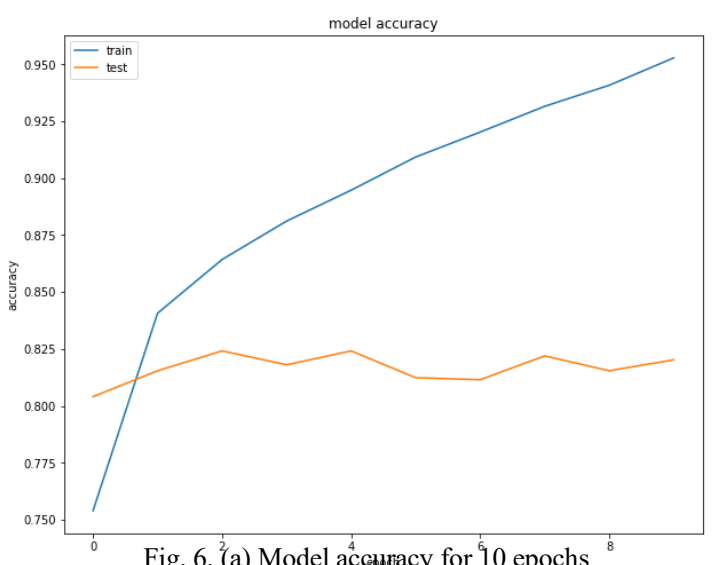

Fig. 6. (a) Model accuracy for 10 epochs

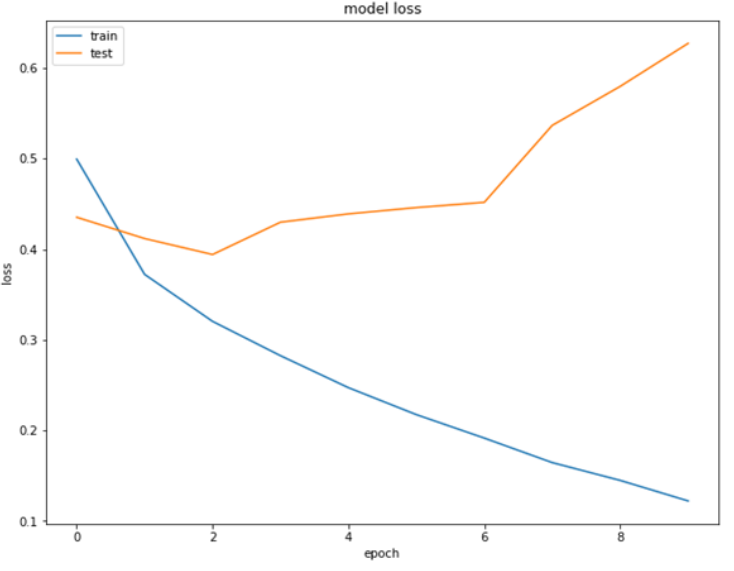

Fig. 6. (b) Model loss for 10 epochs 


\begin{tabular}{|l|c|c|c|c|c|c|c|c|c|}
\hline \multirow{2}{*}{ Technique } & \multicolumn{3}{|c|}{ Positive } & \multicolumn{3}{c|}{ Negative } & \multicolumn{3}{c|}{ Neutral } \\
\cline { 2 - 10 } & Precision & Recall & FI-score & Precision & Recall & FI-score & Precision & Recall & FI-score \\
\hline Naïve Bayes & .55 & .65 & .59 & .80 & .78 & .79 & .73 & .68 & .70 \\
\hline Decision Tree & .73 & .65 & .69 & .83 & .89 & .86 & .78 & .76 & .77 \\
\hline LSTM & .74 & .69 & .69 & .79 & .76 & .77 & .75 & .73 & .74 \\
\hline BiLSTM & .76 & .78 & .78 & .84 & .89 & .87 & .79 & .81 & .80 \\
\hline
\end{tabular}

\section{TWITTER DATA MINING AND VISUALIZATION}

The problem of forecasting civil unrest events such as protests, demonstration and riots has become an important area of interest for researchers. However, due to large volume of data and the dynamic nature of social media platforms, predicting events becomes overwhelmingly impractical. The volume of content being posted on these sites makes it challenging for security analysts to discover such patterns manually. Big data and social media have come together to create a novel field of study called social media mining[15]. Social media mining is the process of representing, analyzing, and extracting actionable patterns and trends from social media data. We have also applied data mining to our Twitter data set containing protest related tweets and tried to extract some interesting patterns and predictions. Fig.7. shows common keywords by number of occurrences extracted from our data set. From these keywords, two locations India Gate and Red Fort were most mentioned where protest were likely to occur. Fig. 8 shows a Twitter account, kept anonymous, exhibiting multiple characteristics associated with inauthentic and suspicious behaviour. Fig.9. shows tweet count per day from 1 January 2021 to 2 February 2021. From the graph it can be seen that tweet count related to farmers protest unexpectedly showed a steep rise from 20 January 2021 to 26 January 2021 indicating an adverse law and order situation. Fig.10. shows the word cloud which is the visual representation of most commonly occurring words or hashtags.

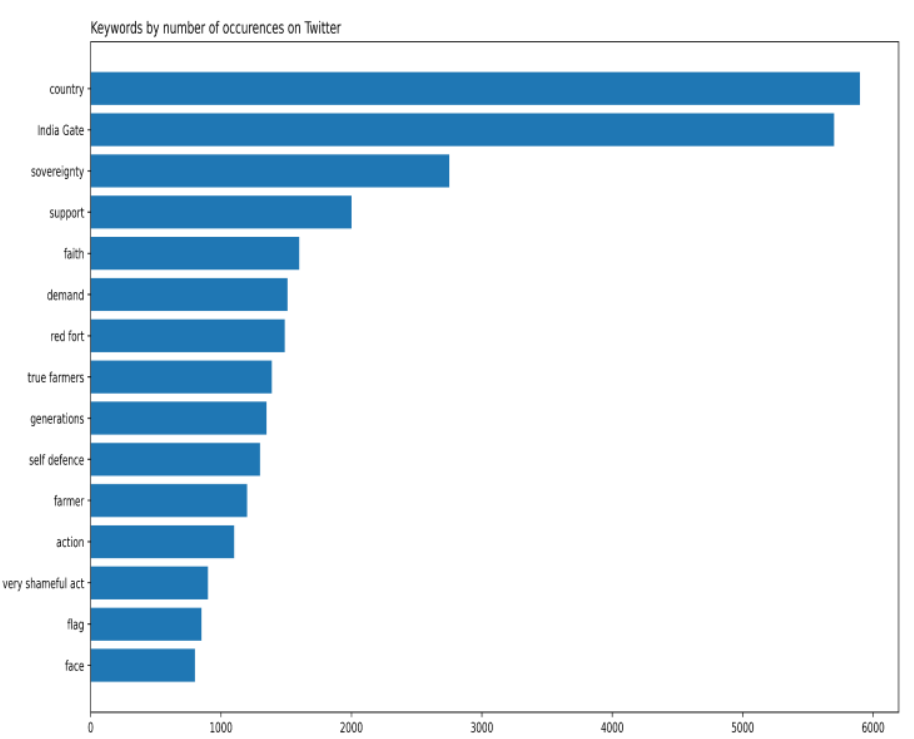

Fig. 7. Keywords by number of occurrences on Twitter

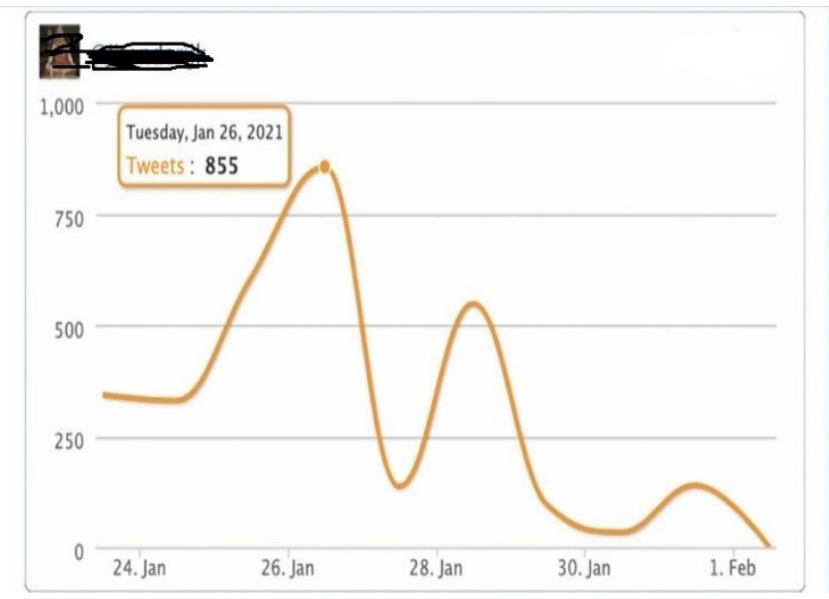

Fig. 8. Twitter Account associated with inauthentic and suspicious behaviour.

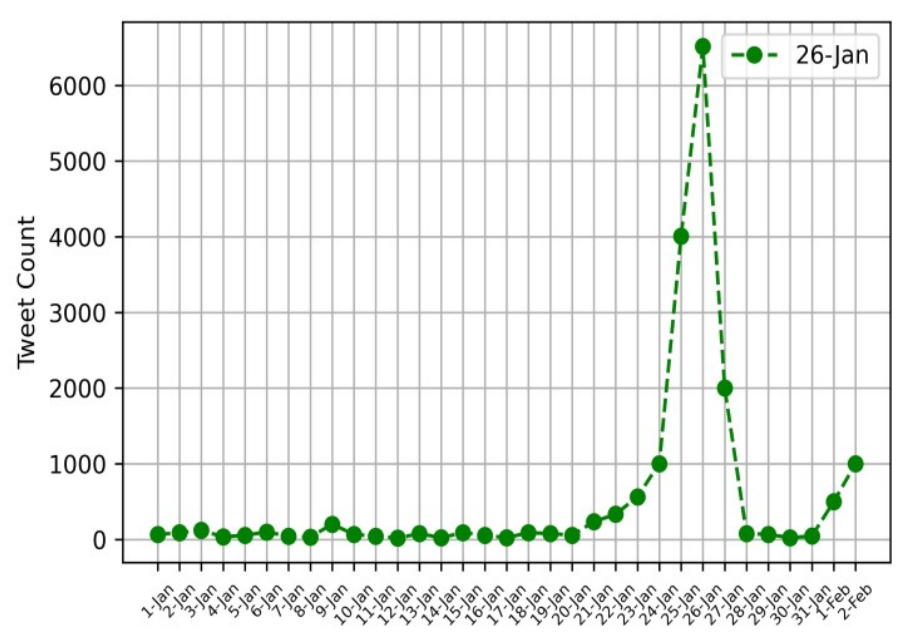

Fig. 9. Tweet count per day for 33 days

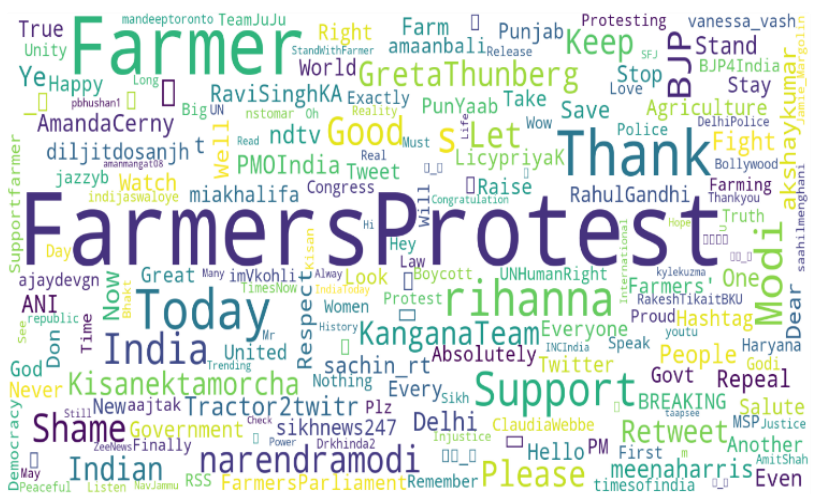

Fig. 10. Word cloud related to Farmers Protest 


\section{CORRELATION AND REGRESSION ANALYSIS}

To test our hypothesis, we applied logistic regression[16] to determine the correlation between protest related tweets and actual protest occurrence. Logistic regression was chosen because actual protests were coded as a binary values. We then applied the fitted model to estimate probability of the protest for a certain time period. Our next step was to determine the probability of protest for a specific period of time(here 33 days) using our fitted model. The generalized results are given below in Fig. 11.

\begin{tabular}{|c|c|c|c|c|c|c|}
\hline \multicolumn{7}{|c|}{ Generalized Linear Model Regression Results } \\
\hline Dep. Variable: & \multicolumn{2}{|c|}{ Actual } & \multicolumn{4}{|c|}{ No. Observations: 33} \\
\hline Model: & \multicolumn{3}{|c|}{ GLM } & \multicolumn{2}{|c|}{ Df Residuals: } & 31 \\
\hline Model Family: & \multicolumn{3}{|c|}{ Binomial } & \multicolumn{2}{|c|}{ Df Model: } & 1 \\
\hline Link Function: & \multicolumn{3}{|l|}{ logit } & \multicolumn{2}{|c|}{ Scale: } & 1.0000 \\
\hline Method: & \multicolumn{3}{|l|}{ IRLS } & \multicolumn{2}{|c|}{ Log-Likelihood: } & -10.599 \\
\hline Date: & \multicolumn{3}{|c|}{ Sun, 03 Oct 2021} & \multicolumn{2}{|c|}{ Deviance: } & 21.197 \\
\hline Time: & \multicolumn{3}{|c|}{$15: 25: 28$} & \multicolumn{2}{|c|}{ Pearson chi2: } & 33.5 \\
\hline No. Iterations: & 5 & & & & & \\
\hline \multicolumn{7}{|c|}{ Covariance Type: robust } \\
\hline coef & std er & $z$ & $\mathbf{P}$ & {$[0.025$} & 0.975] & \\
\hline Intercept -2.4535 & 0.665 & -3.692 & 0.000 & $0-3.756$ & -1.151 & \\
\hline Count 0.0005 & 0.000 & 1.705 & 0.088 & 88.15 & 0.001 & \\
\hline
\end{tabular}

Fig. 11. Generalized linear Model regression results

Since $\mathrm{p}>0.05$ we fail to reject null hypothesis. We conclude that publicly available data on Twitter has potential power to predict occurrence of major protests.

TABLE II. PERFORMANCE RESULTS OF THE MODEL

\begin{tabular}{|l|l|l|l|l|}
\hline $\begin{array}{l}\text { Predicted } \\
\text { Date }\end{array}$ & 25 Jan 2021 & 26 Jan 2021 & 28 Jan 2021 & 2 Feb 2021 \\
\hline TPR & $54.84 \%$ & $100 \%$ & $63.33 \%$ & $100 \%$ \\
\hline TNR & $87.68 \%$ & $91.33 \%$ & $83.61 \%$ & $92.40 \%$ \\
\hline Accuracy & $79 \%$ & $89 \%$ & $80 \%$ & $88 \%$ \\
\hline
\end{tabular}

Table II presents the performance result of logistic regression model. True Positive Rate (TPR), measures the accuracy in predicting the protests, while True Negative Rate (TNR), measures the accuracy in predicting no protests, and the accuracy is calculated as weighted average of TPR and TNR.

\section{CONCLUSION}

In this study, we presented a deep learning based BiLSTM model for predicting civil unrest and protests from Twitter data and tweeting sentiments of its users. We chose Twitter because it is one of the most popular microblogging site and can provide answers to many research questions[17]. The findings of our experiment shows that our model performs better than machine learning models and other deep learning models achieving an accuracy of $82.02 \%$ on testing data and $95.28 \%$ on training data. Further, we tried to validate our proposed model using correlation and regression analysis. We concluded that publicly available data on Twitter has potential power to predict occurrence of major protests when deep learning and data mining is applied to its raw data. Overall ,the aim of the research is to study the role of Twitter in scenario of evolving events such as protests and riots.

\section{REFERENCES}

[1] Rhea Mahajan and Vibhakar Mansotra "Correlating Crime and Social Media: Using Semantic Sentiment Analysis" International Journal of Advanced Computer Science and Applications(IJACSA), 12(3), 2021. http://dx.doi.org/10.14569/IJACSA.2021.0120338

[2] A.Sanjay, K. Anilkumar, R. Krishn, and Y. K. Dwivedi, "International Journal of Information Management Data Insights Sentiment analysis and classification of Indian farmers ' protest using twitter data," Int. J. Inf. Manag. Data Insights, vol. 1, no. 2, p. 100019,2021 .

[3] https://www.thehindu.com/news/national/centres-move-to-repeal farm-laws-reactions/article37573452.ece

[4] R. Compton, C. Lee, T.-C. Lu, L. De Silva, and M. Macy, "Detecting future social unrest in unprocessed Twitter data: "emerging phenomena and big data,", 2013 IEEE International Conference on Intelligence and Security Informatics, 2013.

[5] R. Compton, C. Lee, J. Xu, L. Artieda-Moncada, T.-C. Lu, L. D. Silva, and M. Macy, "Using publicly visible social media to build detailed forecasts of civil unrest," Security Informatics, vol. 3, no. 1, 2014.

[6] J. Cadena, G. Korkmaz, C. J. Kuhlman, and A. Marathe, "Forecasting Social Unrest Using Activity Cascades," October, 2015. https://doi.org/10.1371/journal.pone.0128879.

[7] J. Borge-holthoefer, A. Rivero, Y. Moreno, and S. Gonza, "The Dynamics of Protest Recruitment through an Online Network," pp. 17, 2011.

[8] R. Korolov, D. Lu, J. Wang, G. Zhou, C. Bonial, C. Voss, L. Kaplan, W. Wallace, J. Han, and H. Ji, "On predicting social unrest using social media," 2016 IEEE/ACM International Conference on Advances in Social Networks Analysis and Mining (ASONAM), 2016

[9] F. Qiao, P. Li, X. Zhang, Z. Ding, J. Cheng, and H. Wang, "Predicting social unrest events with Hidden Markov models using GDELT," Discrete Dynamics in Nature and Society, vol. 2017, pp. 113, 2017.

[10] M. Bahrami, Y. Findik, B. Bozkaya, and S. Balcisoy, "Twitter reveals: Using Twitter analytics to predict public protests," arXiv.org, 01-May-2018. [Online]. Available: https://arxiv.org/abs/1805.00358. [Accessed: 06-Dec-2021]

[11] L. Dencik, A. Hintz, and Z. Carey, "Prediction, pre-emption and limits to dissent: Social Media and Big Data uses for policing protests in the United Kingdom," New Media \& Society, vol. 20, no. 4, pp. 1433-1450, 2017.

[12] R. Mahajan and V. Mansotra, "Predicting Geolocation of Tweets : Using Combination of CNN and BiLSTM," Data Sci. Eng., no. 0123456789, 2021.

[13] L. Alfantoukh and A. Durresi, "Techniques for collecting data in social networks", International Conference on Network-Based Information Systems NBIS, vol. 20, pp. 336-341, 2014.

[14] A. Graves and J. Schmidhuber, "Framewise Phoneme Classification with Bidirectional LSTM and Other Neural Network Architectures," Neural Networks, vol. 18,no. 5-6, pp. 602-610, June/July 2005.

[15] D. R. Cox, "The regression analysis of binary sequences," Journal of the Royal Statistical Society. Series B (Methodological), pp. 215242,2012

[16] M. A. Russell, Mining the Social Web. OReilly, 2nd ed., October 2013.

[17] K. K. Kapoor, K. Tamilmani, N. P. Rana, P. Patil, Y. K. Dwivedi, and S. Nerur, "Advances in Social Media Research : Past, Present and Future," pp. 531-558, 2018 
